\title{
Role of spin-lattice coupling in the ultrafast demagnetization of $\mathbf{G d}_{1-x} \mathbf{T b}_{x}$ alloys
}

\author{
A. Eschenlohr, ${ }^{1,2,{ }^{*}}$ M. Sultan, ${ }^{1, *},{ }^{*}$ A. Melnikov,${ }^{3}$ N. Bergeard,,${ }^{1, \ddagger}$ J. Wieczorek, ${ }^{1}$ T. Kachel, ${ }^{2}$ \\ C. Stamm, ${ }^{2, \S}$ and U. Bovensiepen ${ }^{1, \|}$ \\ ${ }^{1}$ Fakultät für Physik and Center for Nanointegration (CENIDE), Universität Duisburg-Essen, Lotharstrasse 1, 47048 Duisburg, Germany \\ ${ }^{2}$ Institut für Methoden und Instrumentierung der Forschung mit Synchrotronstrahlung, Helmholtz-Zentrum Berlin für Materialien und Energie \\ GmbH, Albert-Einstein-Strasse 15, 12489 Berlin, Germany \\ ${ }^{3}$ Abteilung für Physikalische Chemie, Fritz-Haber-Institut der Max-Planck Gesellschaft, Faradayweg 4-6, 14195 Berlin, Germany
}

(Received 10 December 2013; revised manuscript received 11 April 2014; published 27 June 2014)

\begin{abstract}
After excitation by femtosecond laser pulses, Gd and Tb exhibit ultrafast demagnetization in two steps, with the time constant of the second step linked to the coupling strength of the $4 f$ magnetic moments to the lattice. In time-resolved magneto-optical Kerr effect measurements of $\mathrm{Gd}_{1-x} \mathrm{~Tb}_{x}$ alloys, we observe a decrease in this time constant from 33 to 9 ps with Tb content $x$ increasing from 0 to 0.7 . We explain this behavior by the stronger spin-lattice coupling of $\mathrm{Tb}$ compared to $\mathrm{Gd}$, which increases the effective spin-lattice coupling in $\mathrm{Gd}_{1-x} \mathrm{~Tb}_{x}$ with $x$. In contrast, the faster time constant of the first demagnetization step exhibits no dependence on $x$. Additional time- and element-resolved $\mathrm{x}$-ray magnetic circular dichroism measurements show a two-step demagnetization of $\mathrm{Gd}$ and $\mathrm{Tb}$ in $\mathrm{Gd}_{0.6} \mathrm{~Tb}_{0.4}$ with an equivalent time scale of the second step but a different magnitude of demagnetization which persists for $15 \mathrm{ps}$. We explain this by an increased coupling of the Gd $4 f$ magnetic moments to the lattice compared to pure $\mathrm{Gd}$, via interatomic exchange coupling to the neighboring $\mathrm{Tb}$ $4 f$ moments mediated by $5 d$ electrons, which has limited efficiency and allows an estimation of a characteristic time scale of the interatomic exchange coupling. We assign the first demagnetization step to the dynamics of the laser-excited $5 d$ electrons, while the second demagnetization step is dominated by the strength of spin-lattice coupling of the $4 f$ electrons.
\end{abstract}

DOI: 10.1103/PhysRevB.89.214423

PACS number(s): 75.50.Cc, 75.78.Jp, 75.30.Et

\section{INTRODUCTION}

Ultrafast demagnetization of ferromagnetic metals after femtosecond laser excitation, which was first observed in 1996 [1], is an intriguing phenomenon which has only been incompletely understood so far, despite a large body of experimental and theoretical work on the subject (see [2,3] and references therein). The central question here is how spin angular momentum can be transferred in such an ultrafast way, and to which reservoir. An obvious candidate would be spin-lattice coupling, which, however, has been found to occur on a much slower time scale on the order of tens to hundreds of picoseconds [4,5]. Consequently, the microscopic origin of subpicosecond demagnetization is currently under debate. Spin-flip scattering processes such as an Elliott-Yafet type of electron-phonon scattering [6], electron-magnon [7], and electron-electron scattering [8,9] have been proposed, as well as ultrafast magnon emission [10]. Recent work also indicates the importance of spin transport in the superdiffusive regime for ultrafast demagnetization [11-15].

In order to find the relation between ultrafast demagnetization and the microscopic properties of magnetic materials relevant for a theoretical description thereof, such as

\footnotetext{
* Both authors contributed to this work by comparable experimental efforts.

${ }^{\dagger}$ Present address: Nanoscience Department, National Centre for Physics, Islamabad, Pakistan.

${ }^{\ddagger}$ Present address: IPCMS, 23 rue du Loess, BP 43, 67034 Strasbourg

Cedex 2, France.

${ }^{\S}$ Present address: Department of Materials, ETH Zurich, CH-8093

Zurich, Switzerland.

"uwe.bovensiepen@uni-due.de
}

atomic magnetic moment and exchange coupling strength, the ability to vary these properties systematically can give valuable information. Here we investigate the effect of such variations by alloying $\mathrm{Gd}$ and $\mathrm{Tb}$ as two ferromagnetic elements. Several recent studies of ultrafast magnetization dynamics have been performed on magnetic materials consisting of more than one element, i.e. ferri- and ferromagnetic alloys. $\mathrm{Gd}_{25} \mathrm{Fe}_{65.6} \mathrm{Co}_{9.4}$ showed decoupled dynamics of the $\mathrm{Gd}$ and Fe sublattices during ultrafast magnetization reversal and a transient ferromagnetic alignment of the initially antiferromagnetically coupled sublattices [16]. Such a transient ferromagnetic alignment has recently also been seen in ferrimagnetic $\mathrm{Tb}_{16} \mathrm{Fe}_{75} \mathrm{Co}_{9}$ [17]. In $\mathrm{Ni}_{80} \mathrm{Fe}_{20}$, a delay in the demagnetization of Ni compared to $\mathrm{Fe}$ has been observed, which was increased by admixture of $\mathrm{Cu}[18]$.

In this article we focus on the coupling between the spin and lattice degrees of freedom via the orbital anisotropy, which plays a central role in the description of angular momentum transfer between the magnetization and the lattice. We aim to investigate the relation between spin-lattice coupling and ultrafast demagnetization by employing rare earth $\mathrm{Gd}_{1-x} \mathrm{~Tb}_{x}$ alloys as a model system. In our recent study [19], we clarified the role of spin-lattice coupling in ultrafast magnetization dynamics of pure rare earths: In $\mathrm{Gd}$ and $\mathrm{Tb}$, a two-step demagnetization was observed experimentally, in which an initial subpicosecond demagnetization with a time constant of 750 fs for both Gd and Tb was followed by a second, slower reduction of the magnetization. The first demagnetization step occurs on a nonequilibrium time scale, before equilibration between electrons and lattice has taken place [20]. A complex nonequilibrium response of the exchange-split band structure of pure $\mathrm{Gd}$ was observed recently on the same time scale [21]. Only the time constant of the second 
step, corresponding to quasiequilibrium demagnetization after electron-phonon equilibration, was found to be linked to the strength of spin-lattice coupling in the respective rare earth, which depends on the anisotropy of the $4 f$ orbitals. In $\mathrm{Tb}$, a time constant of 8 ps agrees with the strong spin-lattice coupling originating from the anisotropic orbital configuration for an orbital moment of $L=3$ due to the $4 f^{8}$ electron configuration. Gd demagnetized with a time constant of $40 \mathrm{ps,}$ which we explained by the weaker spin-lattice coupling due to the $4 f^{7}$ electron configuration resulting in $L=0$ [22]. In rare earth compounds, long range magnetic order arises from indirect exchange coupling of the $4 f$ electrons, which carry the largest part of the atomic magnetic moment, via the $5 d$ electrons, i.e., RKKY coupling [23]. This coupling is rather strong, which is corroborated by a concomitant demagnetization of $4 f$ and $5 d$ magnetic moment contributions in $\mathrm{Gd}$ [24]. Note that the remaining weak spin-lattice coupling in $\mathrm{Gd}$ is also mediated by the $5 d$ electrons [25]. In $\mathrm{Tb}$ atoms with an orbital quantum number of $L=3$, the $4 f$ magnetic moments exhibit considerably stronger coupling to the lattice than the $4 f$ moments of $\operatorname{Gd}(L=0)$, which can only couple indirectly via the $5 d$ electrons, as discussed above and shown schematically in Fig. 1(b). By alloying Gd and Tb we thus continuously modify the effective strength of spin-lattice coupling according to the alloy composition while staying in an ordered ferromagnetic phase, as will be discussed below.

Here we report on complementary experiments employing the time-resolved magneto-optical Kerr effect (MOKE) in the visible wavelength range and time- and element-resolved $\mathrm{X}$-ray magnetic circular dichroism (XMCD) measurements on $\mathrm{Gd}_{1-x} \mathrm{~Tb}_{x}$ alloys. MOKE allows us to probe concentration dependent magnetization dynamics of the $5 d$ electrons [compare Fig. 1(a)] over a wide range of alloy compositions. We find a continuous decrease of the time constant of the second demagnetization step from 33 to 9 ps with the $\mathrm{Tb}$ content increasing from 0 to 0.7 , which we assign to a spin-lattice coupling increasing with the $\mathrm{Tb}$ fraction. In contrast, the time constant of the first demagnetization step is found to (a)

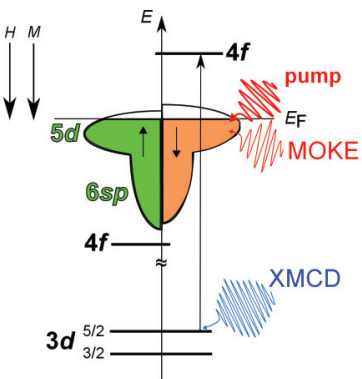

(b)

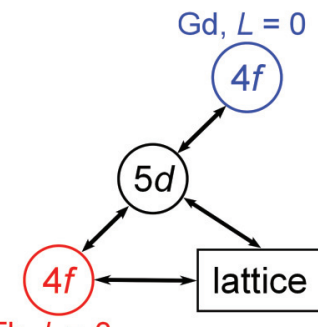

$\mathrm{Tb}, L=3$
FIG. 1. (Color online) (a) Schematic electronic density of states for rare earth elements $\mathrm{Gd}$ and $\mathrm{Tb} .1 .5 \mathrm{eV}$ pump pulses excite predominantly $5 d$ electrons in the vicinity of the Fermi level $E_{\mathrm{F}}$, which are probed by time-resolved MOKE at the same photon energy. $\mathrm{XMCD}$ at the $M_{5}$-edge probes the $4 f$ magnetic moments directly and element selectively by resonantly exciting the $3 d_{5 / 2}$ core level electrons to the unoccupied $4 f_{\downarrow}$ states. $\uparrow$ and $\downarrow$ denote majority and minority spins, while $H$ and $M$ refer to the external magnetic field and magnetization, respectively. (b) The coupling between Gd and $\mathrm{Tb} 4 f$ and $5 d$ magnetic moments and the lattice in $\mathrm{Gd}_{1-x} \mathrm{~Tb}_{x}$ alloy is shown schematically. be uncorrelated to the amount of Tb in the alloy. With XMCD, we distinguish the dynamics of the $\mathrm{Gd}$ and $\mathrm{Tb} 4 f$ magnetic moments by employing a resonant core level excitation at the $M_{5}$ edges as a probe [see Fig. 1(a)]. With this element-selective probe we find a shared slower time constant of the $\mathrm{Gd}$ and $\mathrm{Tb} 4 f$ moments in $\mathrm{Gd}_{0.6} \mathrm{~Tb}_{0.4}$. Thus, the second step of demagnetization of $\mathrm{Gd}$ in the alloy is accelerated compared to pure Gd. Therefore, the Gd $4 f$ magnetic moments in the GdTb alloy experience a stronger coupling to the lattice compared to the pure metal. We estimate the angular momentum transfer from the $4 f$ shell to the lattice, and between $\mathrm{Gd}$ and $\mathrm{Tb}$ in the alloy with a rate model, which allows us to attribute a transiently different magnitude of demagnetization between $\mathrm{Gd}$ and $\mathrm{Tb}$ to a limited efficiency of interatomic exchange coupling mediated by the $5 d$ electrons.

\section{EXPERIMENTAL RESULTS}

\section{A. Concentration dependence of ultrafast demagnetization}

The magneto-optical Kerr effect (MOKE) has been used to investigate the concentration dependence of the ultrafast demagnetization of $\mathrm{Gd}_{1-x} \mathrm{~Tb}_{x}$ thin films for a Tb content $x$ of $0 \leqslant$ $x \leqslant 0.7$. Static and time-resolved MOKE measurements were performed at $790 \mathrm{~nm}$ wavelength in longitudinal configuration, i.e., with an external magnetic field oriented in the sample plane and parallel to the plane of incidence of the laser beam.

Epitaxial, $20 \mathrm{~nm}$ thick $\mathrm{Gd}_{1-x} \mathrm{~Tb}_{x}$ films with (0001) orientation were prepared in ultrahigh vacuum at a base pressure $<10^{-10}$ mbar by coevaporation from electron beam-heated Gd and $\mathrm{Tb}$ sources on W(110) at room temperature. After deposition, the samples were annealed at $700 \mathrm{~K}$ for $10 \mathrm{~min}$ to get smooth epitaxial films. The static magnetic properties of our samples were analyzed by hysteresis loops at base temperatures of $220 \mathrm{~K}$ or higher, as at these temperatures the sample's magnetization could be reversed with the magnetic field of a maximum $0.05 \mathrm{~T}$ available in the experimental chamber [compare Fig. 2(a)]. In Fig. 2(b) the change in magnetization with temperature, as measured by the Kerr rotation, is displayed. The Curie temperature $T_{\mathrm{C}}$ was estimated from the point where the normalized magnetization $M / M_{0}$ is about to vanish. As can be seen in the inset of Fig. 2(b), the resulting values of $T_{\mathrm{C}}$ of the GdTb alloys decrease linearly with increasing $\mathrm{Tb}$ content from $293 \mathrm{~K}$ for pure $\mathrm{Gd}$ to $250 \mathrm{~K}$ for $\mathrm{Gd}_{0.3} \mathrm{~Tb}_{0.7}$, in accordance with literature [23]. The existence of a well-defined $T_{\mathrm{C}}$ demonstrates that our samples are homogeneous ferromagnetic alloys. Furthermore, the change in the shape of the hysteresis loops, going from rectangular for pure Gd to more hard axis like for high Tb content, see Fig. 2(a), originates from the in-plane magnetic anisotropy introduced by $\mathrm{Tb}$ : The W(110) substrate was oriented in such a way that the easy axis of the rare earth film was set perpendicular to the optical plane. This means that in the longitudinal geometry used here the external magnetic field is applied along the hard axis. For pure Gd with no in-plane magnetic anisotropy the axes parallel and perpendicular to the optical plane are equivalent and the sample surface is thus an easy plane. With increasing $x$, the anisotropy of $\mathrm{Gd}_{1-x} \mathrm{~Tb}_{x}$ grows, changing the shape of the hysteresis loops. The hysteresis loops in Fig. 2(a) also show an increased coercivity for alloys with higher $\mathrm{Tb}$ 


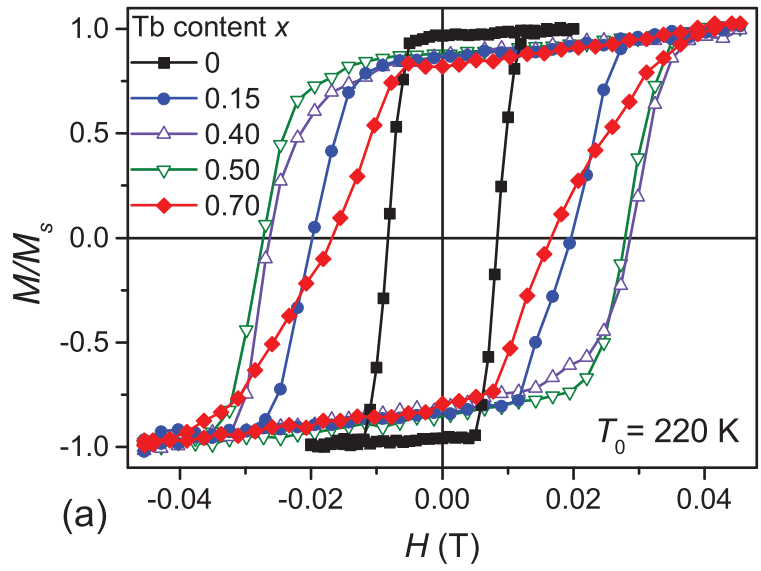

(b)

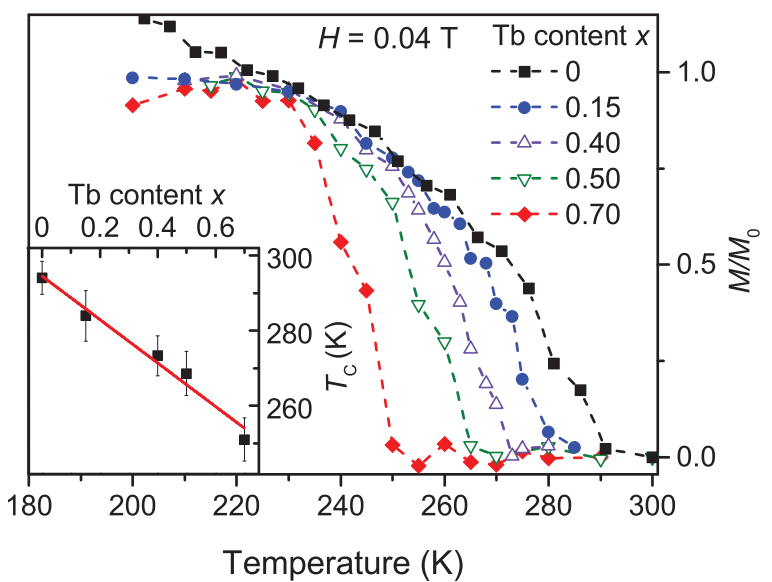

FIG. 2. (Color online) Characterization of the magnetic properties of epitaxial $\mathrm{Gd}_{1-x} \mathrm{~Tb}_{x}$ films on $\mathrm{W}(110)$ by static MOKE. (a) Magnetization $M$, normalized to the saturation magnetization $M_{S}$, depending on the external magnetic field $H$, measured at a base temperature $T_{0}$ of $220 \mathrm{~K}$. Increasing coercivity and/or changing shape of the hysteresis loops with increasing $\mathrm{Tb}$ content point to the influence of the strong $\mathrm{Tb}$ spin-lattice coupling. (b) Temperaturedependent magnetization $M$, normalized to its value $M_{0}$ at $220 \mathrm{~K}$. The inset shows the linear concentration dependence of the Curie temperature $T_{\mathrm{C}}$. The line is a guide to the eye.

content up to $x=0.5$. This effect is consistent with the higher magnetocrystalline anisotropy of $\mathrm{Tb}$, caused by the stronger spin-lattice coupling of its $4 f$ magnetic moments compared to $\mathrm{Gd}$ [26]. These static measurements indicate a stronger effective spin-lattice coupling of $\mathrm{Gd}_{1-x} \mathrm{~Tb}_{x}$ with increasing $\mathrm{Tb}$ content. Next, we investigate how the concentrationdependent modification of the spin-lattice coupling affects the magnetization dynamics of the GdTb alloys.

Femtosecond time-resolved pump-probe experiments were performed using a cavity-dumped Ti:sapphire oscillator with a central wavelength of $790 \mathrm{~nm}$, generating $35 \mathrm{fs}$ pulses at a repetition rate of $1.52 \mathrm{MHz}$. For both the pump and probe beams the fundamental wavelength was employed, with a pump-probe intensity ratio of $4: 1$. Pump and probe beams impinge on the sample almost collinearly, at an angle of about $45^{\circ}$ with respect to the sample normal. All time-resolved MOKE measurements shown in the following were performed at an absorbed pump laser fluence of $1 \mathrm{~mJ} / \mathrm{cm}^{2}$. The base temperature was set to $220 \mathrm{~K}$, which was required to ensure magnetization reversal of the epitaxial film with its high magnetic anisotropy energy due to the Tb constituent [27]. The Kerr rotation was determined by polarization analysis of the reflected probe beam via a Wollaston prism and a balanced diode scheme. To obtain the MOKE signal, the external magnetic field of $0.04 \mathrm{~T}$ was reversed at each point in the pump-probe delay scan, thus reversing the sample's magnetization. The difference between the signals for opposite magnetization directions measured in closed and open phase of the chopper placed in the pump beam, gives the Kerr rotation of the probe beam polarization $\theta_{0}$ and $\theta$ before and after excitation, respectively, which is proportional to the transient magnetization. To exclude errors in the detector calibration, we use the normalized quantity $\Delta \theta / \theta_{0}=\theta / \theta_{0}-1$ to characterize relative pump-induced variations of the magnetization.

The results of our time-resolved MOKE analysis for different compositions of $\mathrm{Gd}_{1-x} \mathrm{~Tb}_{x}$ are displayed in Fig. 3(a). For all compositions, the transient Kerr rotation shows an ultrafast drop of the magnetization within the first few picoseconds after laser excitation, followed by a second, slower magnetization reduction before the magnetization recovers back to its initial value on the time scale of a few hundred picoseconds. Thus, demagnetization of GdTb alloys occurs on two distinct time scales as observed in thin polycrystalline $\mathrm{Gd}$ and $\mathrm{Tb}$ films [19] and epitaxially grown Gd on W(110) [28]. The demagnetization curves of $\mathrm{Gd}_{1-x} \mathrm{~Tb}_{x}$ change strongly with increasing $\mathrm{Tb}$ content. The change of magnetization, proportional to $\Delta \theta / \theta_{0}$, reached after about $200 \mathrm{ps}$, i.e., after equilibration of the spin system and the lattice, is getting larger with $x$. This can be explained in terms of the static temperature-dependent behavior of the magnetization, which is reasonable at this delay: Since $T_{\mathrm{C}}$ of $\mathrm{Gd}_{1-x} \mathrm{~Tb}_{x}$ is reduced with increasing $x$ [compare Fig. 2(b)], bringing the same amount of energy into the ferromagnetic layer by pumping with the same laser fluence and starting at the same base temperature, will, after thermal equilibration, lead to heating increasingly closer to $T_{\mathrm{C}}$, and thus a stronger demagnetization. Afterwards, recovery of the magnetization back to its value before laser excitation occurs on the time scale of heat dissipation out of the ferromagnetic film into the substrate. More importantly, the maximum amount of demagnetization is reached faster for alloys with a higher $\mathrm{Tb}$ content, meaning that the second magnetization drop is accelerated with increasing Tb content.

In order to quantify this dynamics, we determine the corresponding time constants of demagnetization by fitting the time-dependent MOKE data from Fig. 3(a) with a double exponential decay of the form

$$
\frac{\Delta \theta(t)}{\theta_{0}}=S(t)\left[C_{0}+\frac{\Delta M_{1}}{M}\left(1-e^{-t / \tau_{1}}\right)+\frac{\Delta M_{2}}{M}\left(1-e^{-t / \tau_{2}}\right)\right],
$$

where the indices 1 and 2 refer to the first and second step in the demagnetization, respectively, $\frac{\Delta M}{M}$ is the amplitude, $\tau$ is the time constant, and $S(t)$ is a step function at time zero. $C_{0}$ is introduced to describe a steplike change in the Kerr rotation during the first 150 fs observed previously [29]. In the present article we focus on the subsequent reduction of the magnetization. The resulting time constant $\tau_{2}$ for the second magnetization decrease shows a strong change when 

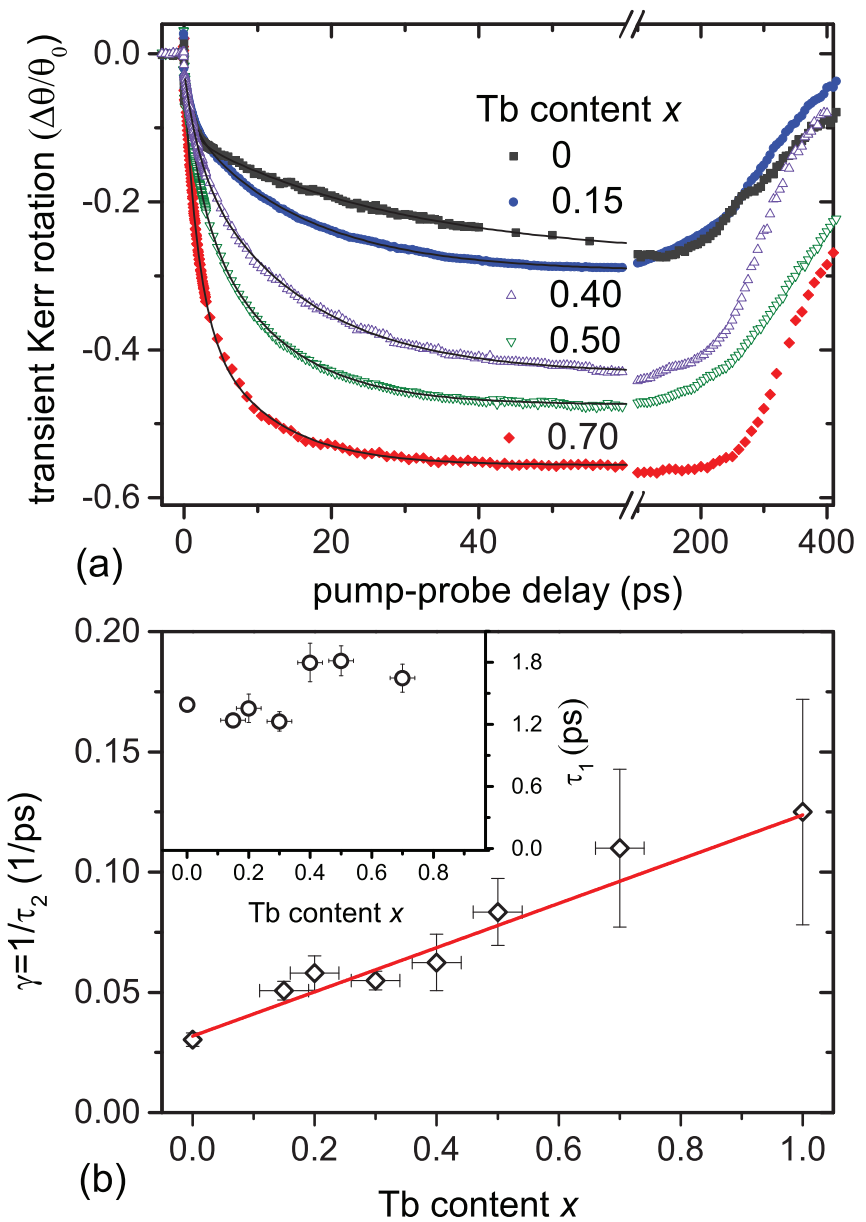

FIG. 3. (Color online) Dependence of ultrafast magnetization dynamics on the $\mathrm{Tb}$ concentration $x$ in $\mathrm{Gd}_{1-x} \mathrm{~Tb}_{x}$ alloy measured with time-resolved MOKE at a base temperature of $220 \mathrm{~K}$ and in an external magnetic field of $\pm 0.04 \mathrm{~T}$. (a) The transient change of the magnetization, as measured by the change in Kerr rotation $\Delta \theta$ normalized to the value $\theta_{0}$ for the unexcited sample, is displayed for five different $\mathrm{Tb}$ concentrations ranging from 0 (uppermost curve) to 0.7 (lowermost curve). (b) The resulting rate of the quasiequilibrium process $\gamma=1 / \tau_{2}$, with $\tau_{2}$ being the time constant of the second step of demagnetization as derived from double exponential fits to the time-resolved MOKE data [plotted as black lines in (a)], shows an increase towards larger $\mathrm{Tb}$ concentration. This behavior is well described by a linear fit to the data (solid line). $\gamma$ for pure Tb is taken from [19]. Inset: In the time constant $\tau_{1}$ of the first step of demagnetization, no clear trend with increasing $\mathrm{Tb}$ concentration can be observed.

going from pure $\mathrm{Gd}$ to $\mathrm{Gd}_{0.85} \mathrm{~Tb}_{0.15}$, from 33 to $20 \mathrm{ps,} \mathrm{which}$ indicates that the spin-lattice coupling of $\mathrm{Tb}$ dominates the magnetization dynamics in the alloys on longer time scales already for a rather low $\mathrm{Tb}$ content. Moreover, we see a continuous decrease in the time constant for the second demagnetization step when the Tb content is increased further. The rate of this quasiequilibrium process $\gamma=1 / \tau_{2}$ [see Fig. 3(b)] can be fitted with a linear dependence, including the value previously found for pure $\mathrm{Tb}$ and linked to its strong, direct $4 f$-lattice coupling [19]. We can thus attribute the accelerated demagnetization to an increased effective spin-lattice coupling in GdTb with increasing Tb content.
The time constants $\tau_{1}$ of the first magnetization drop vary between 1.2 and 1.8 ps, as shown in the inset of Fig. 3(b). These time constants are larger than the time constant of about 750 fs observed previously on pure, epitaxially grown Gd [28] at a base temperature of $50 \mathrm{~K}$. This difference in the time constants is due to the different base temperatures in [28] and the measurement presented here. A similar increase from about 800 fs to 1.2 ps when raising the temperature from 80 to $220 \mathrm{~K}$ has previously been observed on pure $\mathrm{Gd}$ and attributed to an increased contribution of phonon-mediated spin-flip scattering processes to ultrafast demagnetization when going above the Debye temperature [29]. Coming back to the concentration dependence in $\mathrm{Gd}_{1-x} \mathrm{~Tb}_{x}, \tau_{1}$ does not show any particular trend when the alloy composition is varied, in striking contrast to $\tau_{2}$. The fact that $\tau_{2}$ exhibits a clear dependence on the spin-lattice coupling strength represents the main result of our concentration dependent time-resolved MOKE measurements.

The question remains whether only $\mathrm{Tb}$ in $\mathrm{Gd}_{1-x} \mathrm{~Tb}_{x}$ contributes to the accelerated demagnetization for higher $\mathrm{Tb}$ content. The Gd and $\mathrm{Tb} 4 f$ moments are coupled through the interatomic exchange mediated by $5 d$ electrons and $4 f-5 d$ intra-atomic coupling [see Fig. 1(b) and Sec. I]. It can thus be expected that also the Gd $4 f$ moments in a GdTb alloy show an enhanced coupling to the lattice which is then mediated by the interatomic exchange coupling to neighboring $\mathrm{Tb}$ atoms. Element-sensitive XMCD measurements were therefore employed in order to disentangle the individual contributions of the $\mathrm{Gd}$ and $\mathrm{Tb} 4 f$ magnetic moments in the alloy, which will be described in the next section.

\section{B. Element-resolved ultrafast magnetization dynamics}

$\mathrm{X}$-ray magnetic circular dichroism (XMCD) is the difference of absorption of circularly polarized $\mathrm{x}$ rays in a ferroor ferrimagnetic sample for parallel and antiparallel alignment of the sample's magnetization with respect to the x-ray propagation direction [30]. Since XMCD involves an electronic transition from a core level to the unoccupied $4 f_{\downarrow}$ states in $\mathrm{Gd}$ and $\mathrm{Tb}$ [compare Fig. 1(a)], it is an element-specific probe, which allows us to look at the elemental constituents of $\mathrm{GdTb}$ separately. With XMCD at the $M_{5,4}$ edges, we directly probe the localized $4 f$ magnetic moments of $\mathrm{Gd}$ and $\mathrm{Tb}$ in the alloy and complement the magneto-optical analysis above. Static and time-resolved XMCD measurements were performed at the BESSY II storage ring operated by Helmholtz Zentrum Berlin für Materialien und Energie GmbH, Berlin, Germany.

For XMCD measurements in transmission geometry, $10 \mathrm{~nm}$ thick polycrystalline $\mathrm{Gd}_{0.6} \mathrm{~Tb}_{0.4}$ samples were prepared by co-evaporation from $\mathrm{Gd}$ and $\mathrm{Tb}$ sources on $500 \mathrm{~nm}$ thick freestanding $\mathrm{Al}$ foils under UHV conditions at a base pressure $<10^{-10}$ mbar and at room temperature [31]. Y buffer and cap layers of 50 and $5 \mathrm{~nm}$ thickness, respectively, were employed to protect the rare earth film against degradation. The $\mathrm{Gd}$ and $\mathrm{Tb} 4 f$ magnetic moments in the alloy show ferromagnetic alignment, as can be seen from the negative (positive) XMCD at both $M_{5}\left(M_{4}\right)$ absorption edges in the static XMCD spectra in Fig. 4. The base temperature for the XMCD analysis was set to $82 \mathrm{~K}$, well below the Curie temperature of about $270 \mathrm{~K}$ for this alloy composition, see Fig. 2(b) and [23]. Furthermore, $\mathrm{Gd}$ and $\mathrm{Tb}$ exhibit identical hystereses in the alloy; an external 

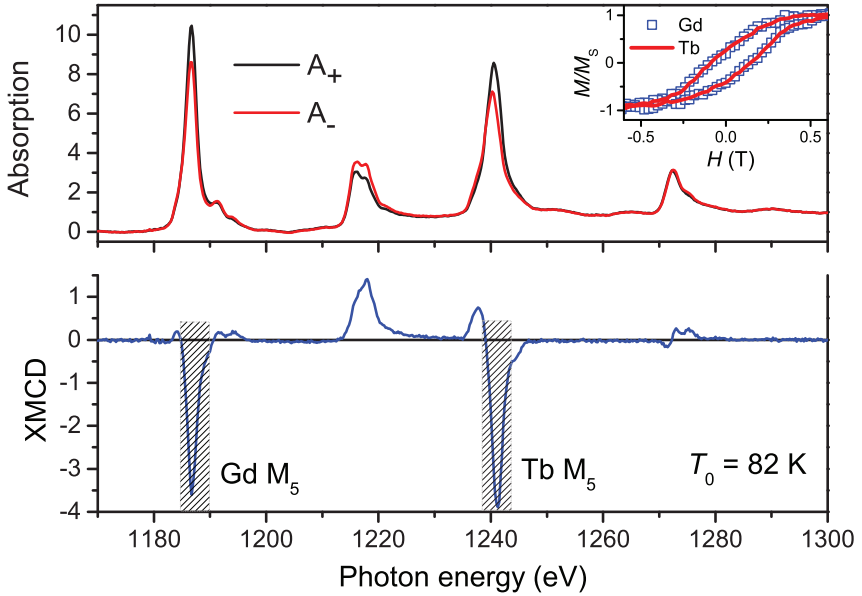

FIG. 4. (Color online) X-ray absorption spectra of $\mathrm{Gd}_{0.6} \mathrm{~Tb}_{0.4}$ at the $\mathrm{Gd}$ and $\mathrm{Tb} M_{5,4}$ edges measured with circularly polarized $\mathrm{x}$ rays, with the sample's magnetization oriented parallel $\left(\mathrm{A}_{+}\right)$and antiparallel ( $\mathrm{A}_{-}$) to the $\mathrm{x}$-ray propagation direction by applying an external magnetic field $H= \pm 0.5 \mathrm{~T}$, are displayed in the upper panel. The absorption spectra are normalized to the continuum step after the $\mathrm{Tb} M$ edges. The lower panel shows the XMCD, which is proportional to $\mathrm{A}_{-}-\mathrm{A}_{+}$, with the proportionality factor [32] given by the angle of $35^{\circ}$ to the surface normal under which $H$ is applied and the x-ray polarization degree of $90 \%$. The shaded areas mark the photon energies where the time-resolved XMCD measurements were performed. The inset depicts element-resolved hysteresis loops of $\mathrm{Gd}$ and $\mathrm{Tb}$, taken at the respective $M_{5}$ edges.

magnetic field of $\pm 0.5 \mathrm{~T}$ applied along the $\mathrm{x}$-ray propagation direction is sufficient to saturate the sample's magnetization, as can be seen in the inset of Fig. 4. Since the magnetization of the $\mathrm{Gd}_{0.6} \mathrm{~Tb}_{0.4}$ sample is oriented preferentially in plane [33], and XMCD measures the projection of the $4 f$ magnetic moments along the X-ray propagation direction, the angle between the sample normal and the $\mathrm{x}$-ray propagation direction was set to $35^{\circ}$ for all static and time-resolved XMCD measurements.

For the time- and element-resolved measurements of the magnetization dynamics, the x-ray energy was fixed to the value for the maximum dichroic signal at the $M_{5}$ edge of $\mathrm{Gd}$ at $1186 \mathrm{eV}$, or respectively to $1241 \mathrm{eV}$ for $\mathrm{Tb}$. At each step in the pump-probe delay, the XMCD signal was obtained by reversing the external magnetic field applied to the sample. Circularly polarized, 100 fs short x-ray probe pulses were provided by the BESSY II Femtoslicing source [34,35]. In the Femtoslicing process, an intense femtosecond laser pulse modulates the energy of a part of an electron bunch circulating in the storage ring, allowing this ultrashort "slice" of electrons to be split off from the main bunch and radiate a correspondingly short X-ray pulse in a subsequent insertion device. At the BESSY II source, $x$ rays are generated at a repetition rate of $6 \mathrm{kHz}$, provided by a first Ti:sapphire amplifier, while the pump pulses are generated from a second amplifier running at $3 \mathrm{kHz}$, seeded by the same Ti:sapphire oscillator. In the pump-probe measurements we thus alternate between pump-probe and probe only, leading to a high sensitivity towards pump-induced dynamics. For exciting the sample, 50 fs laser pulses with $780 \mathrm{~nm}$ wavelength and an incident fluence of $3.4 \mathrm{~mJ} / \mathrm{cm}^{2}$ were used. Due to the jitter-free synchronization of the laser

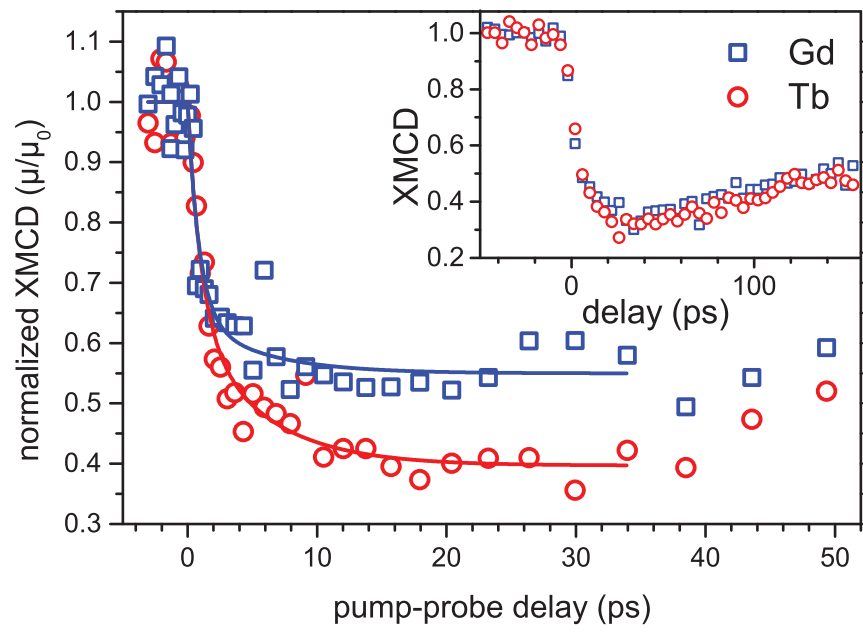

FIG. 5. (Color online) Time- and element-resolved ultrafast demagnetization of $\mathrm{Gd}$ (squares) and $\mathrm{Tb}$ (circles) in $\mathrm{Gd}_{0.6} \mathrm{~Tb}_{0.4}$. The temporal evolution of the XMCD signal, which corresponds to the transient, element-resolved $4 f$ magnetic moment $\mu$, normalized to its value $\mu_{0}$ before laser excitation, is shown for a pump-probe delay of up to 50 ps. XMCD acquired with 10 ps long x-ray pulses is displayed in the inset. All XMCD measurements were performed at base temperature $82 \mathrm{~K}$ and in an external magnetic field of $\pm 0.5 \mathrm{~T}$.

pump pulses and x-ray probe pulses, which are generated from the same source, i.e., Ti:sapphire oscillator, this setup results in a total time resolution of 130 fs for the time-resolved XMCD measurements [35].

As can be seen in Fig. 5, a reduction of both the $\mathrm{Gd}$ and $\mathrm{Tb} 4 f$ magnetic moments in the alloy is observed after laser excitation. The main figure shows data with 130 fs time resolution. For the inset, longer $\mathrm{x}$-ray pulses with a duration of $10 \mathrm{ps}$ full width at half maximum, which are provided in the low- $\alpha$ mode of the BESSY II storage ring [36], were employed. This dataset shows that the recovery of the magnetic moments of both constituents on a time scale of several hundred picoseconds proceeds simultaneously. The initial drop in the magnetic moments is broadened in time here due to the X-ray pulse duration of $10 \mathrm{ps}$ [36] and potentially some additional electronic jitter between pump and probe which does not occur in Femtoslicing mode. The dataset with 130 fs time resolution reveals that the demagnetization happens in two steps, with the first, subpicosecond step comprising about half of the total magnitude of demagnetization. This initial drop results in a relative change of the magnetic moment $\mu / \mu_{0}$ of $\mathrm{Gd}$ and $\mathrm{Tb}$, respectively, to about 0.7 , where $\mu_{0}$ represents the equilibrium value of the magnetic moment before optical excitation. Subsequently, the magnetic moment of $\mathrm{Tb}$ is reduced to about $0.4 \mu_{0}$ during the next $20 \mathrm{ps}$, before recovery sets in at a longer time scale. The dynamic behavior of $\mathrm{Gd}$ in the alloy follows that of Tb. Interestingly, the magnitude of demagnetization is slightly lower than for $\mathrm{Tb}$.

In order to analyze these experimental results in a more quantitative manner, the demagnetization curves were fitted with a double exponential decay of the form

$$
\mu(t)=\mu_{0}+S(t)\left[\Delta \mu_{1}\left(1-e^{-t / \tau_{1}}\right)+\Delta \mu_{2}\left(1-e^{-t / \tau_{2}}\right)\right],
$$


TABLE I. Overview of demagnetization time constants of pure $\mathrm{Gd}, \mathrm{Tb}$, and $\mathrm{Gd}_{1-x} \mathrm{~Tb}_{x}$ alloy. The element-averaged time constants for $\mathrm{Gd}_{0.6} \mathrm{~Tb}_{0.4}$ from our MOKE analysis are somewhat different from those found in our XMCD measurements due to the different base temperatures in these experiments, as discussed in the main text.

\begin{tabular}{lcc}
\hline \hline & $\tau_{1}(\mathrm{ps})$ & $\tau_{2}(\mathrm{ps})$ \\
\hline $\mathrm{Gd} \mathrm{[4]}$ & - & $100 \pm 80$ \\
$\mathrm{Gd} \mathrm{[41]}$ & - & 48 \\
$\mathrm{Gd} \mathrm{[19]}$ & $0.76 \pm 0.25$ & $40 \pm 10$ \\
$\mathrm{Gd} \mathrm{[28]}$ & 0.70 & - \\
$\mathrm{Gd}[21]$ & $0.86 \pm 0.10$ & - \\
$\mathrm{Gd}$ in $\mathrm{Gd}_{0.6} \mathrm{~Tb}_{0.4}$ (this work) & $0.89 \pm 0.29$ & $5.3 \pm 2.3$ \\
$\mathrm{~Tb}$ in $\mathrm{Gd}_{0.6} \mathrm{~Tb}_{0.4}$ (this work) & $1.03 \pm 0.25$ & $5.3 \pm 2.3$ \\
$\mathrm{Gd}_{0.6} \mathrm{~Tb}_{0.4}(\mathrm{MOKE}$ this work) & $1.80 \pm 0.19$ & $16 \pm 3$ \\
$\mathrm{~Tb}[19]$ & $0.74 \pm 0.25$ & $8 \pm 3$ \\
\hline \hline
\end{tabular}

where $S(t)$ refers to a step function at time zero, $\mu_{0}$ is the initial XMCD value at negative pump-probe delay, i.e., the magnetic moment under equilibrium conditions, and $\tau_{i}$ and $\Delta \mu_{i}, i=1,2$ are the time constant and amplitude of the first femtosecond and second picosecond exponential decay, respectively. In order to facilitate a comparison, we list the time constants $\tau_{1}$ and $\tau_{2}$ for pure $\mathrm{Gd}, \mathrm{Tb}$, and $\mathrm{Gd}_{0.6} \mathrm{~Tb}_{0.4}$ as known from literature and this study in Table I. For the first step of demagnetization, the double exponential fit results in characteristic time constants of $0.89 \pm 0.29$ ps for $\mathrm{Gd}$ and $1.03 \pm 0.25$ ps for $\mathrm{Tb}$ in $\mathrm{Gd}_{0.6} \mathrm{~Tb}_{0.4}$. These time constants agree within error bars with those found in pure $\mathrm{Gd}$ and $\mathrm{Tb}$ [19] (compare Table I). The time constants for $\mathrm{Gd}_{0.6} \mathrm{~Tb}_{0.4}$ measured with XMCD are both shorter than the element-averaged one derived from our MOKE measurements for the same alloy composition, which is $1.8 \pm 0.2 \mathrm{ps}$. This deviation is due to the different equilibrium temperatures for MOKE and XMCD measurements of 220 and $82 \mathrm{~K}$ discussed in [29] and above.

Regarding the second step of demagnetization, a common time constant of $5.3 \pm 2.3$ ps for $\mathrm{Gd}$ and $\mathrm{Tb}$ is obtained. The second step in the demagnetization of $\mathrm{Gd}_{0.6} \mathrm{~Tb}_{0.4}$ thus occurs with a time constant that is slightly smaller [37] but comparable to that of pure $\mathrm{Tb}(8 \pm 3 \mathrm{ps}$, compare Table I and [19]). Therefore, the demagnetization of Gd in GdTb alloy is clearly faster than that of pure $\mathrm{Gd}$, which shows a time constant of $40 \pm 10$ ps [19] (see also Table I). Consequently, the accelerated demagnetization of $\mathrm{Gd}$ in $\mathrm{Gd}_{60} \mathrm{~Tb}_{40}$ confirms an enhanced coupling of the Gd $4 f$ magnetic moments to the lattice via $5 d$ electron mediated interatomic exchange coupling to the neighboring $\mathrm{Tb}$ atoms, which represents the main result of our time- and element-resolved XMCD measurements.

The origin of the difference in the relative magnitude of demagnetization for $\mathrm{Gd}$ and $\mathrm{Tb}$ in $\mathrm{Gd}_{0.6} \mathrm{~Tb}_{0.4}$ between 5 and 40 ps, see Fig. 5, remains to be discussed [38]. As will be discussed below, this difference is a consequence of the different spin-lattice coupling of Gd and Tb, cf. Fig. 1(b). It furthermore leads to an estimation of the characteristic time scale of the interatomic exchange coupling mediated by the $5 d$ electrons and the respective magnetic moment transfer rate. The scheme of angular momentum transfer indicated in Fig. 1(b) already suggests that in a quasiequilibrium process the demagnetization of the $\mathrm{Tb} 4 f$ shell must be stronger than that of the Gd $4 f$ shell to provide a significant flow of angular momentum from Gd $4 f$ to $\mathrm{Tb} 4 f$ through the $5 d$ electrons. In this light, the observed smaller demagnetization of the Gd $4 f$ shell appears to be reasonable. In the following we describe the angular momentum transfer processes during the second step of demagnetization in $\mathrm{Gd}_{1-x} \mathrm{~Tb}_{x}$ with a simple rate model in order to account for our observations from the concentration-dependent and element-resolved experiments.

\section{DISCUSSION}

\section{A. The characteristic time scale of the RKKY exchange interaction}

In order to further narrow down the microscopic mechanisms behind the second demagnetization step observed in $\mathrm{Gd}_{1-x} \mathrm{~Tb}_{x}$, we estimate the amount of angular momentum transferred between the localized $4 f$ magnetic moments, the $5 d$ electrons, and the collective excitations of the lattice [39], as shown schematically in Fig. 1(b). Here we expand on a similar estimate performed in our previous study on pure $\mathrm{Gd}$ and $\mathrm{Tb}$ [19] by now taking concentration dependent changes in $\mathrm{Gd}_{1-x} \mathrm{~Tb}_{x}$, as well as angular momentum transfer between the Gd and Tb magnetic moments into account.

We first look at the element-averaged angular momentum transfer rate $\gamma$ from the $\mathrm{Gd}$ and $\mathrm{Tb}$ magnetic moments to the lattice, which changes with the Tb concentration $x$, as seen in our time-resolved MOKE measurements [compare Fig. 3(b)]. In a quasiequilibrium demagnetization process, which is taking place on the second, slower demagnetization step observed here in $\mathrm{Gd}_{1-x} \mathrm{~Tb}_{x}$, the change in magnetization with time is given at the beginning of this quasiequilibrium process by $d m /\left.d t\right|_{t \approx 0}=-\gamma \cdot \Delta m$. Here $\Delta m$ represents the relative change of magnetization within the second demagnetization step in units of the saturated moment $\mu_{S}=x \mu_{\mathrm{Tb}}^{0}+$ $(1-x) \mu_{\mathrm{Gd}}^{0}$, with $\mu_{\mathrm{Tb}}^{0}=9.34 \mu_{\mathrm{B}}$ and $\mu_{\mathrm{Gd}}^{0}=7.55 \mu_{\mathrm{B}}$. It is determined by the extent of demagnetization achieved during the first, nonequilibrium step, before the quasiequilibrium demagnetization sets in, and the final magnetic moment reached at the end of the process. We suppose for simplicity that the transient temperature stays constant, i.e., without heat dissipation out of the laser-excited sample volume. Moreover, $\Delta m$ is assumed to depend on the sample composition due to the $x$-dependent $T_{\mathrm{C}}$, and on experimental conditions like sample temperature, absorbed energy, and cooling conditions. Here $\Delta m$ ranges between 0.2 and 0.3 , as obtained from double exponential fits to our MOKE data according to Eq. (1). Similar values were obtained in earlier experiments [19]. We will thus use $\Delta m=0.25$ for estimations below.

We base our estimate on a linear approximation of the concentration dependent trend in the rate of the quasiequilibrium demagnetization process $\gamma(x)=a+b x$ with $a=0.033 \mathrm{ps}^{-1}$ and $b=0.097 \mathrm{ps}^{-1}$, where $a$ and $b$ were obtained from the linear fit of $\gamma(x)$, see Fig. 3(b). We link this linear dependence to the different pathways for angular momentum transfer to the lattice by assuming $\gamma(x)=\gamma_{i}+\gamma_{d} x \alpha(x)$, where $\gamma_{d}$ and $\gamma_{i}$ are the rates of direct angular momentum transfer from the $4 f$ shell to the lattice occurring on $\mathrm{Tb}$ ions, and indirect transfer through $5 d$ electrons on both $\mathrm{Tb}$ and Gd ions, respectively [19]. The latter is supposed identical for both types of ions owing to 
the similarity of ion masses and the shared $5 d$ electron band of $\mathrm{Gd}$ and $\mathrm{Tb}$, so that this contribution does not depend on the Tb content $x$. Since the factor $\alpha(x)=\mu_{\mathrm{Tb}}^{0} / \mu_{S}(x)$ is close to unity, giving only a small correction to the linear dependence of $\gamma$ on $x$, we neglect it in the following. We thus end up with $\gamma_{i} \approx 0.03 \mathrm{ps}^{-1}$ and $\gamma_{d} \approx 0.1 \mathrm{ps}^{-1}$. From this we estimate quasiequilibrium rates of angular momentum transfer to the lattice per $\mathrm{Gd}$ and $\mathrm{Tb}$ ion to $\sigma_{\mathrm{Gd}}=\gamma_{i} \mu_{\mathrm{Gd}}^{0} \Delta m \approx 0.06 \mu_{\mathrm{B}} / \mathrm{ps}$ and $\sigma_{\mathrm{Tb}}=\left(\gamma_{i}+\gamma_{d}\right) \mu_{\mathrm{Tb}}^{0} \Delta m \approx 0.3 \mu_{\mathrm{B}} / \mathrm{ps}$, respectively, which is in good agreement with our previous study [19].

So far we only considered the angular momentum transfer from the $\mathrm{Gd}$ and $\mathrm{Tb}$ ions to the lattice while implicitly assuming that both ions exhibit identical dynamics during the quasiequilibrium demagnetization, which is motivated by the results of our MOKE experiments sensitive to the element-averaged magnetization of the GdTb alloys. The angular momentum transfer to the lattice in the alloy is in this case characterized by the average transfer rate $\sigma_{\mathrm{GdTb}}=$ $x \sigma_{\mathrm{Tb}}+(1-x) \sigma_{\mathrm{Gd}}$, which is found to be $\approx 0.16 \mu_{\mathrm{B}} / \mathrm{ps}$ for $x=0.4$. Such an identical dynamics of the relative magnetic moments of the $\mathrm{Gd}$ and $\mathrm{Tb}$ ions, i.e., $m_{G d}=\mu_{\mathrm{Gd}} / \mu_{\mathrm{Gd}}^{0}$ and $m_{\mathrm{Tb}}=\mu_{\mathrm{Tb}} / \mu_{\mathrm{Tb}}^{0}$ could be the result of $5 d$ electron mediated interatomic exchange coupling between neighboring ions (RKKY coupling). However, in fact, this coupling competes with the interaction with the lattice. As shown below, the equilibration rate of $\mathrm{Tb}$ and $\mathrm{Gd}$ magnetic moments allows an estimation of the characteristic time scale of the interatomic exchange interaction. The interatomic angular momentum transfer rate via $5 d$ electron mediated exchange coupling from one $\mathrm{Gd}$ ion to all neighboring $\mathrm{Tb}$ ions is, according to the values determined above, $\sigma_{\mathrm{Gd}}^{\mathrm{RKKY}}=\sigma_{\mathrm{GdTb}}-\sigma_{\mathrm{Gd}} \approx 0.10 \mu_{\mathrm{B}} / \mathrm{ps}$ and correspondingly to one $\mathrm{Tb}$ ion from all neighboring $\mathrm{Gd}$ ions $\sigma_{\mathrm{Tb}}^{\mathrm{RKKY}}=\sigma_{\mathrm{Tb}}-\sigma_{\mathrm{GdTb}} \approx 0.14 \mu_{\mathrm{B}} / \mathrm{ps}$. Furthermore, we need to consider the number of nearest neighbors between whom angular momentum can be transferred. Thus, in the hcp lattice with coordination number $N_{C}=12$ formed by $\mathrm{Gd}_{1-x} \mathrm{~Tb}_{x}$, the following expressions also hold: $\sigma_{\mathrm{Gd}}^{\mathrm{RKKY}}=x N_{C} \sigma^{\mathrm{RKKY}}$ and $\sigma_{\mathrm{Tb}}^{\mathrm{RKY}}=(1-x) N_{C} \sigma^{\mathrm{RKKY}}$, where $\sigma^{\mathrm{RKKY}}$ is the angular momentum transfer rate via $5 d$ electron mediated interatomic exchange coupling between neighboring $\mathrm{Gd}$ and $\mathrm{Tb}$ ions. We suppose $\mathrm{Gd}$ and $\mathrm{Tb}$ in $\mathrm{Gd}_{1-x} \mathrm{~Tb}_{x}$ to be identical in the considered interatomic coupling mechanism, as they share the $5 d$ band. Combining the above expressions, we obtain $\sigma^{\mathrm{RKKY}}=$ $\Delta m\left[\mu_{\mathrm{Tb}}^{0} \gamma_{d}+\left(\mu_{\mathrm{Tb}}^{0}-\mu_{\mathrm{Gd}}^{0}\right) \gamma_{i}\right] / N_{C} \approx 0.02 \mu_{\mathrm{B}} / \mathrm{ps}$. This means that while assuming identical dynamics of the $\mathrm{Gd}$ and $\mathrm{Tb}$ magnetic moments, the value of the interatomic angular momentum transfer rate $\sigma^{\mathrm{RKKY}}$ is about $0.02 \mu_{\mathrm{B}} / \mathrm{ps}$ under the present experimental conditions. On the other hand, in the quasiequilibrium time window, the dynamics of the $\mathrm{Gd}$ and Tb ions cannot be absolutely identical: A difference in the relative change in magnetization of $\mathrm{Gd}$ and $\mathrm{Tb}$ will trigger a nonzero flux of angular momentum from one sublattice to another in order to obtain thermal equilibrium. In the picture discussed here, this difference originates from the stronger coupling of the $\mathrm{Tb} 4 f$ magnetic moments to the lattice, so that the magnetization of the Tb sublattice is reduced faster than that of Gd. The value of this difference is as larger as stronger the coupling of $\mathrm{Tb}$ ions to the lattice, and as smaller as larger the maximum possible interatomic transfer rate $\sigma_{\max }^{\mathrm{RKKY}}$. Since the latter quantity is a universal characteristic of rare earth metals, the interesting remaining question is an estimation of $\sigma_{\max }^{\mathrm{RKK}}$.

Our time- and element-resolved XMCD measurements show that the demagnetization at the $\mathrm{Gd}$ and $\mathrm{Tb}$ ions in $\mathrm{Gd}_{0.6} \mathrm{~Tb}_{0.4}$ is indeed identical in terms of the time scale $\tau_{2}$ (compare Table I). However, in Fig. 5 one can also realize the difference $\Delta \mu / \mu_{0}$ in the normalized XMCD, which corresponds to a relative change of the magnetic moment, of $\mathrm{Gd}$ and $\mathrm{Tb}$ in $\mathrm{Gd}_{0.6} \mathrm{~Tb}_{0.4}$. At 5-20 ps the demagnetization of $\mathrm{Gd}$ is smaller than that of $\mathrm{Tb}$, and the difference increases up to $\Delta \mu / \mu_{0} \approx 0.1$ at 20 ps. Subsequently, the transient difference starts to reduce and vanishes at about 40 ps (see also Fig. 5, inset), while the Tb magnetic moment is nearly constant within this interval. Consequently, the characteristic time of the process behind this transient difference can be estimated to be in the range of 5-10 ps. The magnetic moment to be transferred between neighboring $\mathrm{Gd}$ and $\mathrm{Tb}$ ions through $5 d$ electron mediated interatomic exchange coupling, in order to level off their relative magnetic moments, is $\Delta \mu^{\mathrm{RKKY}}=\mu_{\mathrm{Tb}}^{0}\left(\frac{\Delta \mu}{\mu_{0}}\right) /\left\{N_{C}\left[1+x\left(\frac{\mu_{\mathrm{Tb}}^{0}}{\mu_{\mathrm{Gd}}^{0}}-1\right)\right]\right\} \approx 0.07 \mu_{\mathrm{B}}$. Estimating $\sigma_{\max }^{\mathrm{RKKY}}$ with the above characteristic time which is


estimate is nevertheless consistent with $\sigma^{\text {RKKY }}$ obtained from our MOKE experiments and can be treated as a characteristic value limiting the rate of interatomic angular momentum transfer in rare earths.

We conclude that the angular momentum transfer via interatomic exchange interaction mediated by $5 d$ electrons explains the difference in $\mathrm{Tb}$ and $\mathrm{Gd}$ magnetic moments for 5-40 ps observed in Fig. 5. While an additional channel for the transfer of angular momentum from $\mathrm{Gd}$ to the lattice is opened via the neighboring $\mathrm{Tb}$ atoms, the interatomic exchange interaction acts as a bottleneck for the magnetic moment transfer between $\mathrm{Gd}$ and $\mathrm{Tb}$ and limits the loss of magnetic moment of the Gd ions to the observed value.

\section{B. The mechanism of the demagnetization process}

In finding that the first, subpicosecond time scale of demagnetization does not show a signature of a dependence on the strength of coupling of the $4 f$ magnetic moments to the lattice, whereas the second demagnetization time constant clearly does, we confirm our earlier observations regarding the two-step demagnetization of rare earths [19]. Demagnetization on the subpicosecond, nonequilibrium time scale is initiated by the $5 d$ electrons, but the behavior on longer time scales is dominated by the strength of direct $4 f$-lattice coupling and therefore strongly depends on the orbital anisotropy of the $4 f$ shell, which defines the efficiency of angular momentum transfer to the lattice.

Regarding the initial subpicosecond demagnetization, laser-induced changes of the population of the exchange-split $5 d$ band and a reduction of the exchange splitting on a similar time scale of $0.86 \pm 0.10$ ps have been found recently in photoemission experiments [21] (compare Table I). This is another indication that the initial demagnetization is launched by the $5 d$ electrons. Since $\mathrm{Gd}$ and $\mathrm{Tb}$ in $\mathrm{Gd}_{1-x} \mathrm{~Tb}_{x}$ have a shared $5 d$ band, a first step in the demagnetization initiated 
by the optically excited $5 d$ electrons also explains why no dependence of $\tau_{1}$ on the alloy composition was found.

Furthermore, the time constant of 33 ps reported here for the second demagnetization step of pure $\mathrm{Gd}$ is on the same order as the spin-lattice relaxation time observed [4,40] and described theoretically [41] earlier, see Table I. Consequently, a description of the ultrafast demagnetization process by equilibrium properties, in particular the Curie temperature $T_{\mathrm{C}}$, should take the excited state into account on time scales shorter than this spin-lattice relaxation time, as thermal equilibration between the lattice and spin systems has not taken place yet.

This brings us to the question, which theoretical models potentially represent an adequate description of ultrafast magnetization dynamics in rare earths and their alloys? The microscopic three-temperature model (M3TM) [6] proposes electron-phonon spin-flip scattering of the Elliott-Yafet type as the origin of ultrafast demagnetization. It explicitly treats the case of Gd. However, the two steps observed during demagnetization are explained by the same process, namely spin flips, which occur with a certain probability at each electron-phonon scattering event [6]. In the framework of the M3TM, a two-step demagnetization is said to occur when coupling between the spin and orbital degrees of freedom is weak [42]. This is supposed to be the case for rare earths $\mathrm{Gd}$ and $\mathrm{Tb}$, in contrast to $3 d$ ferromagnets [42]. However, the very similar spin-orbit coupling constants of $\mathrm{Gd}$ and $\mathrm{Tb}$ are larger than those of the $3 d$ ferromagnets [43]. This leaves differences in $L$ as a possible cause for weak spinlattice coupling via the spin-orbit interaction. As discussed throughout this article, spin-lattice coupling is certainly weak for $\mathrm{Gd}$ with an orbital moment $L=0$. In contrast, $\mathrm{Tb}$ with $L=3$ and the corresponding anisotropic $4 f$ orbital configuration shows strong spin-lattice coupling, leading to increasing effective spin-lattice coupling with increasing $x$ in $\mathrm{Gd}_{1-x} \mathrm{~Tb}_{x}$, as demonstrated by our analysis of the static magnetic properties above. And yet, $\mathrm{Gd}_{1-x} \mathrm{~Tb}_{x}$ shows a two-step demagnetization. Therefore, our conclusion that two different microscopic processes at the localized moment are responsible for the two steps in the ultrafast demagnetization of rare earths and their alloys suggests amendments to the M3TM [6] taking direct spin-lattice coupling of localized $4 f$ magnetic moments into account. Magnetization dynamics in materials consisting of several sublattices or constituents has furthermore been described phenomenologically by atomistic modeling $[44,45]$, which however neglects direct spin-lattice coupling and can therefore also not be applied to $\mathrm{Tb}$ and its alloys [17]. Consequently, theoretical descriptions of the initial subpicosecond demagnetization $[6,9,11]$ are to be extended to also include spin-lattice coupling and spin-orbit interaction.

\section{CONCLUSION}

We have shown that combined time-resolved MOKE and XMCD measurements give information on the interactions mediating the ultrafast demagnetization process in $\mathrm{Gd}_{1-x} \mathrm{~Tb}_{x}$ rare earth alloys. Concentration-dependent MOKE measurements revealed a two step demagnetization, with the time constant of the first step being independent of $x$, while the second reduction in magnetization was accelerated with increasing $\mathrm{Tb}$ content, in accordance with an increasing coupling of the $4 f$ magnetic moments to the lattice via the anisotropic $4 f$ orbital configuration of Tb. From element-sensitive XMCD measurements we see that the second demagnetization step occurs for $\mathrm{Gd}$ in $\mathrm{Gd}_{0.6} \mathrm{~Tb}_{0.4}$ on the same time scale as for $\mathrm{Tb}$, indicating an enhanced coupling of the Gd $4 f$ moments to the lattice via indirect interatomic exchange (RKKY) coupling to the $\mathrm{Tb} 4 f$ moments. A transient difference in the amount of demagnetization between $\mathrm{Gd}$ and $\mathrm{Tb}$ in $\mathrm{Gd}_{0.6} \mathrm{~Tb}_{0.4}$ is attributed to a limited efficiency of this interatomic exchange coupling. We thus conclude that two different microscopic processes are responsible for the two-step demagnetization typical for rare earths $\mathrm{Gd}, \mathrm{Tb}$, and their alloys. The first, subpicosecond demagnetization is explained by the direct excitation of the $5 d$ valence electrons by the pump laser, while we confirm spin-lattice coupling of the localized $4 f$ magnetic moments as the driving force behind demagnetization on time scales of several picoseconds or longer.

\section{ACKNOWLEDGMENTS}

We thank Rolf Mitzner and Karsten Holldack for support during the time-resolved XMCD measurements, and the Helmholtz Zentrum Berlin for financial support for travel to BESSY II. C.S. thanks the ETH Zurich for financial support during the writing of the manuscript. Financial support by the German Ministry of Education and Research BMBF Grant 05K10PG2 FEMTOSPEX, the Deutsche Forschungsgemeinschaft through project ME3570/1, and the DAAD-HEC Pakistan is gratefully acknowledged.
[1] E. Beaurepaire, J.-C. Merle, A. Daunois, and J.-Y. Bigot, Phys. Rev. Lett. 76, 4250 (1996).

[2] A. Kirilyuk, A. V. Kimel, and Th. Rasing, Rev. Mod. Phys. 82, 2731 (2010).

[3] A. Kirilyuk, A. V. Kimel, and Th. Rasing, Rep. Prog. Phys. 76, 026501 (2013).

[4] A. Vaterlaus, T. Beutler, and F. Meier, Phys. Rev. Lett. 67, 3314 (1991).

[5] A. Vaterlaus, T. Beutler, D. Guarisco, M. Lutz, and F. Meier, Phys. Rev. B 46, 5280 (1992).

[6] B. Koopmans, G. Malinowski, F. Dalla Longa, D. Steiauf, M. Fähnle, T. Roth, M. Cinchetti, and M. Aeschlimann, Nat. Mater. 9, 259 (2009).
[7] E. Carpene, E. Mancini, C. Dallera, M. Brenna, E. Puppin, and S. De Silvestri, Phys. Rev. B 78, 174422 (2008).

[8] M. Krauß, T. Roth, S. Alebrand, D. Steil, M. Cinchetti, M. Aeschlimann, and H. C. Schneider, Phys. Rev. B 80, 180407(R) (2009).

[9] B. Y. Mueller, A. Baral, S. Vollmar, M. Cinchetti, M. Aeschlimann, H. C. Schneider, and B. Rethfeld, Phys. Rev. Lett. 111, 167204 (2013).

[10] A. B. Schmidt, M. Pickel, M. Donath, P. Buczek, A. Ernst, V. P. Zhukov, P. M. Echenique, L. M. Sandratskii, E. V. Chulkov, and M. Weinelt, Phys. Rev. Lett. 105, 197401 (2010).

[11] M. Battiato, K. Carva, and P. M. Oppeneer, Phys. Rev. Lett. 105, 027203 (2010). 
[12] A. Melnikov, I. Razdolski, T. O. Wehling, E. Th. Papaioannou, V. Roddatis, P. Fumagalli, O. Aktsipetrov, A. I. Lichtenstein, and U. Bovensiepen, Phys. Rev. Lett. 107, 076601 (2011).

[13] D. Rudolf et al., Nat. Commun. 3, 1037 (2011).

[14] A. Eschenlohr, M. Battiato, P. Maldonado, N. Pontius, T. Kachel, K. Holldack, R. Mitzner, A. Föhlisch, P. M. Oppeneer, and C. Stamm, Nat. Mater. 12, 332 (2013).

[15] E. Turgut et al., Phys. Rev. Lett. 110, 197201 (2013).

[16] I. Radu et al., Nature (London) 472, 205 (2011).

[17] A. R. Khorsand, M. Savoini, A. Kirilyuk, A. V. Kimel, A. Tsukamoto, A. Itoh, and Th. Rasing, Phys. Rev. Lett. 110, 107205 (2013).

[18] S. Mathias et al., Proc. Natl. Acad. Sci. U.S.A. 109, 4792 (2012).

[19] M. Wietstruk, A. Melnikov, C. Stamm, T. Kachel, N. Pontius, M. Sultan, C. Gahl, M. Weinelt, H. A. Dürr, and U. Bovensiepen, Phys. Rev. Lett. 106, 127401 (2011).

[20] U. Bovensiepen, J. Phys.: Condens. Matter 19, 083201 (2007).

[21] R. Carley, K. Döbrich, B. Frietsch, C. Gahl, M. Teichmann, O. Schwarzkopf, P. Wernet, and M. Weinelt, Phys. Rev. Lett. 109, 057401 (2012).

[22] This difference in spin-lattice coupling is responsible for a two orders of magnitude difference in the magnetoelastic constants of Gd and Tb, see B. Coqblin, The Electronic Structure of RareEarth Metals and Alloys (Academic, London, 1977).

[23] D. M. S. Bagguley, J. P. Partington, J. A. Robertson, and R. C. Woods, J. Phys. F: Met. Phys 10, 967 (1980).

[24] M. Wietstruk, Ph.D. thesis, Technische Universität, Berlin, 2010.

[25] M. Colarieti-Tosti, S. I. Simak, R. Ahuja, L. Nordström, O. Eriksson, D. Åberg, S. Edvardsson, and M. S. S. Brooks, Phys. Rev. Lett. 91, 157201 (2003).

[26] K. Tajima, J. Phys. Soc. Jpn. 31, 441 (1971).

[27] K. Starke, F. Heigl, A. Vollmer, M. Weiss, G. Reichardt, and G. Kaindl, Phys. Rev. Lett. 86, 3415 (2001).

[28] M. Sultan, A. Melnikov, and U. Bovensiepen, Phys. Status Solidi B 248, 2323 (2011).

[29] M. Sultan, U. Atxitia, A. Melnikov, O. Chubykalo-Fesenko, and U. Bovensiepen, Phys. Rev. B 85, 184407 (2012).

[30] J. Stöhr, J. Electron. Spectrosc. Relat. Phenom. 75, 253 (1995).

[31] Please note that the different film thicknesses and substrates for the samples used in our MOKE and XMCD studies do not affect the magnetization dynamics on time scales less than $\approx 50$ ps. Only the time needed for recovery of the magnetic signal back to its value before laser excitation is longer in the XMCD samples on freestanding membranes, due to a less effective heat transfer out of the excited region, see M. Sultan, Ph.D. thesis, Freie Universität, Berlin, 2012.

[32] C. T. Chen, Y. U. Idzerda, H.-J. Lin, N. V. Smith, G. Meigs, E. Chaban, G. H. Ho, E. Pellegrin, and F. Sette, Phys. Rev. Lett. 75, 152 (1995).

[33] A. Berger, A. W. Pang, and H. Hopster, J. Magn. Magn. Mater. 137, L1 (1994).

[34] S. Khan, K. Holldack, T. Kachel, R. Mitzner, and T. Quast, Phys. Rev. Lett. 97, 074801 (2006).

[35] C. Stamm et al., Nat. Mater. 6, 740 (2007).

[36] J. Feikes, K. Holldack, P. Kuske, and G. Wüstefeld, Proceedings of EPAC (European Physical Society Accelerators Group at CERN, Geneva, Switzerland, 2004), p. 1954.

[37] The time constant $\tau_{2}$ for $\mathrm{Gd}$ and $\mathrm{Tb}$ in $\mathrm{Gd}_{0.6} \mathrm{~Tb}_{0.4}$ is potentially somewhat shorter than the $\tau_{2}$ derived for pure $\mathrm{Tb}$ in [19] because we could not take the recovery of the magnetic signal to its equilibrium value before laser excitation into account in fitting the present dataset, in contrast to [19].

[38] A systematic error in the measurement is excluded: During the experiment we frequently alternated between measurements at the $\mathrm{Gd}$ and $\mathrm{Tb} M_{5}$ edges to ensure that potential drifts in the temporal or spatial pump-probe overlap affect both measurements equally.

[39] L. Zhang and Q. Niu, Phys. Rev. Lett. 112, 085503 (2014).

[40] A. Melnikov, H. Prima-Garcia, M. Lisowski, T. Gießel, R. Weber, R. Schmidt, C. Gahl, N. M. Bulgakova, U. Bovensiepen, and M. Weinelt, Phys. Rev. Lett. 100, 107202 (2008).

[41] W. Hübner and K. H. Bennemann, Phys. Rev. B 53, 3422 (1996).

[42] T. Roth, A. J. Schellekens, S. Alebrand, O. Schmitt, D. Steil, B. Koopmans, M. Cinchetti, and M. Aeschlimann, Phys. Rev. X 2, 021006 (2012).

[43] J. Stöhr and H. C. Siegmann, Magnetism: From Fundamentals to Nanoscale Dynamics (Springer, Berlin, 2006).

[44] T. A. Ostler et al., Nat. Commun. 3, 666 (2012).

[45] J. H. Mentink, J. Hellsvik, D. V. Afanasiev, B. A. Ivanov, A. Kirilyuk, A. V. Kimel, O. Eriksson, M. I. Katsnelson, and Th. Rasing, Phys. Rev. Lett. 108, 057202 (2012). 